Authors have nothing to disclose with regard to commercial support.

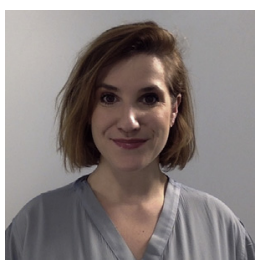

\section{BATTLE OF THE SEXES}

To the Editor:

In passing the portrait photographs of our cardiothoracic colleagues in the corridor, one baseline characteristic is apparent: they are overwhelmingly male. Our experience in the specialty spans from 1 to 8 years, and from our individual viewpoints, we agree that there is a problem with sexism. We also see the environment around us changing, however, with increasing numbers of female surgeons of various grades and with slowly shifting attitudes of acceptance.

We applaud Stephens and Fiedler ${ }^{1}$ for drawing attention to women's plights in this male-dominated specialty. Our initial enthusiasm, however, was rapidly curbed. The quoted gender gap analyses are subjective, illustrating experiences rather than facts. The issue is by its nature qualitative, and the sharing of experiences and solutions is essential to encourage and support. Nonetheless, we need objective assessments of the progress of women through training and their success on the consultant level. More importantly, the "tips" to "improve the success of women resident physicians" are generic and can be applied to every surgical resident, regardless of potential discriminatory traits such as gender, race or sexual orientation.

Let us talk about the actual grit of it: The sexes are different, from leadership styles, to self-analysis, down to the very physical. Bring a "woman's touch" to leadership, and respect is easily lost; bring assertive and driven qualities, and risk being labelled as aggressive. We tread the fine line of "fitting in with the boys" and yet maintaining those qualities that are different and bring variety and excellence to the table. Not to mention physical differences-women taking sick days because of dysmenorrhea, delaying training after childbirth, dedicating their breaks to pumping breast milk.

Gone must be the days of senior surgeons telling their colleagues that "girls shouldn't be surgeons." The attitude

\footnotetext{
The Editor welcomes submissions for possible publication in the Letters to the Editor section that consist of commentary on an article published in the Journal or other relevant issues. Authors should: • Include no more than 500 words of text, three authors, and five references. • Type with double-spacing. • See http://jtcs.ctsnetjournals.org/ misc/ifora.shtml for detailed submission instructions. • Submit the letter electronically via jtcvs.editorialmanager.com. Letters commenting on an article published in the JTCVS will be considered if they are received within 6 weeks of the time the article was published. Authors of the article being commented on will be given an opportunity of offer a timely response ( 2 weeks) to the letter. Authors of letters will be notified that the letter has been received. Unpublished letters cannot be returned.
}

arises from visible and outspoken sexism, but also from the underlying, more insidious, and difficult to pin down unconscious biases that we all possess. Often, the individual is unaware of his or her own unacknowledged preconceptions, making them challenging to tackle. The resulting insult or undermining is often unintended, but it can carry the same weight.

We need to approach these very real facts, normalize physical needs, and to shed light nonconfrontationally on bias and microaggressions. Our field is changing, and we must talk about how to forge an enjoyable, successful career and personal life with allowances for our differences, whether they be in gender, family commitments, or physical ailments.

We therefore wish to tell our (female) colleagues (1) do not be discouraged on the basis of gender; (2) adopt a zero-tolerance policy on sexism, and speak up when you encounter it; and (3) do this while not interpreting every comment or situation as sexist-equally, there may be other reasons that you did not get to perform that last case. We must lead by example-continue doing our jobs, being present at meetings and participating in support networks, highlighting the normality of women in our career. So, let us embrace our differences and focus on "\#WeRAllCTSurgeons"- that way, we may see the day that nobody thinks twice about female cardiothoracic surgeons.

Kathrin Freystaetter, $M D^{a}$ Puwalani Vidanapathirana, MRCS Louise Kenny, FRCS

${ }^{a}$ Department of Cardiothoracic Surgery James Cook University Hospital Middlesbrough, United Kingdom

${ }^{b}$ Department of Cardiothoracic Surgery Freeman Hospital

Newcastle Upon Tyne, United Kingdom

\section{Reference}

1. Stephens EH, Fiedler AG. Beyond \#ILookLikeASurgeon. J Thorac Cardiovasc Surg. 2018;156:1131-6.

https://doi.org/10.1016/j.jtcvs.2019.01.061

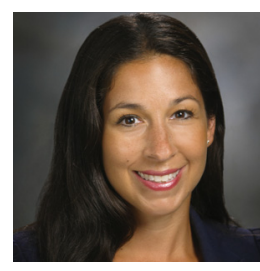

A CALL FOR CEASEFIRE: WE CAN ONLY MOVE FORWARD TOGETHER

Reply to the Editor:

Recent years have demonstrated remarkable strides in the rising numbers of women entering the field of cardiothoracic surgery, with promise for continued growth in the future 
Author has nothing to disclose with regard to commercial support.

with a pipeline of women currently in cardiothoracic surgical training. ${ }^{1,2}$ However, despite the enormous gains that have been made, women remain drastically underrepresented in our field. There are issues that accompany being an underrepresented minority that will persist until we reach a tipping point of cultural change. Moreover, there are additional issues inherent in the differences between the sexes that will always be relevant to our profession, regardless of the quantitative balance of men and women in our field, even if we someday reach completely equal representation.

In "Beyond \#ILookLikeASurgeon," Stephens and Fielder $^{3}$ did an excellent job of summarizing the state of women in cardiothoracic surgery, highlighting important published data addressing gender issues. They subsequently provided a series of tips to overcome obstacles, intended to serve as a systematic approach to supporting and facilitating careers of women and underrepresented minorities. In their letter to the Editor, "Battle of the Sexes," Freystaetter and colleagues ${ }^{4}$ provide critiques to the article by Stephens and Fiedler, ${ }^{3}$ suggesting that their quoted gender gap analyses are subjective and do not include facts. Let us be clear. There exist ample objective, unavoidable data that clearly highlight gender-related differences between men and women in our specialty, many of which were presented by Stephens and Fiedler. ${ }^{3}$ These data have been drawn from studies including quantitative, appropriate analyses of social and behavioral phenomena.

Freystaetter and colleagues ${ }^{4}$ appear to be discounting studies that report on how women feel compared with how men feel (in terms of preparation for practice, opportunities for autonomy, support and mentorship in the workplace, etc), because these outcomes are in the eye of the beholder. Let us look at a clinical correlate: recent years have brought heightened interest and emphasis in examining patientreported outcomes (PROs) in addition to traditional measures of success for our operative procedures. ${ }^{5}$ PROs are incredibly important adjuncts to the existing types of data that we already collect. PROs consist of subjective data points-measurable, quantitative, and valid results-that describe subjective perceptions. Nobody would argue that if a patient has survived an operation but feels awful that there is a problem that needs intervention.

Stephens and Fiedler ${ }^{3}$ did not state that women were less prepared for practice; rather, they provided data that surveyed women felt less prepared. ${ }^{6}$ These findings do not serve as evidence of an ability gap, but rather evidence of a confidence gap, which most certainly merits being acknowledged and addressed. Likewise, Stephens and
Fiedler $^{3}$ shared Meyerson and colleagues' findings ${ }^{7}$ that female trainees did not believe that they achieved operative autonomy as often as male trainees. Clearly, further studies are necessary to determine whether women are truly less autonomous or just feel less autonomous-but the fact that responses differed by gender merits attention. Another recently presented study evaluated male and female graduates of a single training program in the course of 60 years, with the finding that women felt significantly less prepared for their written and oral board examinations despite similar board pass rates. ${ }^{8}$ We do not know whether the women felt less prepared because of differences in their training experiences, or whether they had the exact same experience but felt less confident as a result of beliefs about themselves stemming from internal or external pressures; however, these issues need to be clarified so that they can be fixed. These types of investigations are exactly what we need better to address the unmet needs of women and other underrepresented minorities. The literature that exists may be limited and only show the tip of the iceberg, but it provides a foundation for understanding and a strong impetus to dive deeper.

Freystaetter and colleagues ${ }^{4}$ further point out that the tips shared in "Beyond \#ILikeLookASurgeon" could be applied to all trainees-not just women and minorities. While this is a valid point, it does not negate the importance and particular relevance of these suggestions for the targeted population. One might draw an analogy to the circumstance of providing preoperative counseling to a patient seeking to optimize his or her likelihood for favorable postoperative outcomes. We might encourage the patient to avoid cigarette smoke and to eat a nutritious diet. Certainly, such advice could be accused of being "generic" and applicable to everyone whether or not they are having surgery. Nonetheless, it is still incredibly important for the preoperative patient! Do male cardiothoracic surgeons need mentorship, support networks, and leadership skills? Of course. Their applicability to a greater population, however, does not render these suggestions any less relevant for women and minority individuals seeking to overcome career challenges, and they remain important components of that toolkit.

Women are constituting greater proportions of our workforce year by year. ${ }^{1,2}$ Some of the issues currently faced may improve simply by reaching a tipping point, whereby women are adequately represented to limit sexism and combat unconscious bias. Moreover, as we consider physician burnout, wellness, and needs of all surgeons for work-life balance, we are recognizing that there are unmet needs shared among men and women. We are getting better at discussing parental leave, rather than just maternity leave. And, as insinuated by Freystaetter and colleagues, ${ }^{4}$ everyone needs mentorship. Overall 
cultural changes in our field, along with continuing to increase our numbers, will bring help to many of the problems highlighted by the anecdotes in "Beyond \#ILookLikeASurgeon." ${ }^{3}$ With greater numbers, we will most certainly be able to find proper locker rooms when going on procurements. As we change perceptions of what a cardiothoracic surgeon looks like, inappropriate and sexist comments will hopefully be fewer and further between. However, some problems faced by female cardiothoracic surgeons will persist, regardless of how many of us there are, and these issues were well highlighted by Stephens and Fiedler. ${ }^{3}$ Challenges of childbearing are biologically unique to women and occur disproportionately more often among female cardiothoracic surgeons. ${ }^{9}$ We must recognize that issues such as infertility, pregnancy complications, and lactation needs will always be specific to women. Efforts to support women through such events are vital in acknowledging their role as important contributors to our workforce, and such progress is mandatory to ensuring that the brightest and best trainees comprise the future of our field.

Taken together, Stephens and Fiedler ${ }^{3}$ and Freystaetter and colleagues ${ }^{4}$ provide practical and complementary approaches to enhancing the success of women in cardiothoracic surgery. The big picture messages are more alike than discrepant: Recognize that women and men are not the same. Embrace those differences, so that we can look forward to a time when women are encouraged, enabled, and supported in their pursuit of a career in our specialty.

\section{Mara B. Antonoff, MD University of Texas MD Anderson Cancer Center Houston, Tex}

\section{References}

1. Donington JS, Litle VR, Sesti J, Colson YL. The WTS report on the current status of women in cardiothoracic surgery. Ann Thorac Surg. 2012;94:452-8; discussion 458-9.

2. Antonoff MB, David EA, Donington JS, Colson YL, Litle VR, Lawton JS, et al. Women in thoracic surgery: 30 years of history. Ann Thorac Surg. 2016;101: 399-409.

3. Stephens EH, Fiedler AG. Beyond \#ILookLikeASurgeon. J Thorac Cardiovasc Surg. 2018;156:1131-6.

4. Freystaetter K, VIdanapathirana P, Kenny L. Battle of the sexes. J Thorac Cardiovasc Surg. 2019;157:e393.

5. Khullar OV, Fernandez FG. Patient-reported outcomes in thoracic surgery. Thorac Surg Clin. 2017;27:279-90.

6. Stephens EH, Robich MP, Walters DM, DeNino WF, Aftab M, Tchantchaleishvili V, et al. Gender and cardiothoracic surgery training: specialty interests, satisfaction, and career pathways. Ann Thorac Surg. 2016;102:200-6.

7. Meyerson SL, Sternbach JM, Zwischenberger JB, Bender EM. The effect of gender on resident autonomy in the operating room. J Surg Educ. 2017;74:e111-8.

8. Luc J, Moon M, Antonoff MB. The impact of sex on confidence and perception of training in cardiothoracic surgery. Presented at: The Society of Thoracic Surgeons 55th Annual Meeting, January 27-29, 2019; San Diego, CA.

9. Pham DT, Stephens EH, Antonoff MB, Colson YL, Dildy GA, Gaur P, et al. Birth trends and factors affecting childbearing among thoracic surgeons. Ann Thorac Surg. 2014;98:890-5.

https://doi.org/10.1016/j.jtcvs.2019.01.112

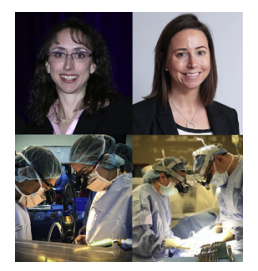

WE HAVE MET THE ENEMY; THE ENEMY IS

US

Reply to the Editor:

We appreciate Freystaetter and colleagues $^{1}$ for their correspondence regarding our editorial. ${ }^{2}$ It is encouraging to see increased dialog about being a minority within our field. Even 5 years ago, proposing to examine potential disparities between minorities and the majority within training or discussing challenges minorities faced was frequently disparaged.

Freystaetter and colleagues ${ }^{1}$ discuss, as we do in our editorial, conscious and unconscious bias, as well as gender differences, including the difficulty of being an effective female leader. We particularly appreciate their call for more robust data with regard to barriers faced by women and minorities in our field, and we wholeheartedly agree that more remains to be done in this area. In response, we highlight here key take-home messages from the current literature regarding women and minorities in surgery, of which all in our field should be aware:

1. A third of women in US academic medical centers reported experiencing sexual harassment and bias (vs $4 \%$ of men).

2. Women in US academic medical centers earn $10 \%$ less, are promoted more slowly, receive fewer honors, and hold fewer leadership positions than men-even after adjusting for age, experience, specialty, rank, and academic and research productivity. ${ }^{4,5}$ People are less likely to hire a woman than a man with identical qualifications; less likely to give credit, including grant support, to a woman for identical accomplishments; and less likely to give the benefit of the doubt to a woman than to a man. ${ }^{5}$

3. Women thoracic surgical trainees described being given significantly less "meaningful autonomy," given less access to career advice, given less effective mentorship and sponsorship, and feeling less ready for practice then men. ${ }^{6}$ This discrimination becomes more acute with seniority.

We as a specialty are well positioned to use these objective data to make impactful changes, starting at the top. In 2006 the National Academies of Science advised all major societies to ensure that their podium, editorial, and leadership boards reflect their diverse membership. Cardiothoracic surgery has heeded this call, with formal professional career development programs, such as The American Association for Thoracic Surgery Cardiothoracic Careers College and Leadership Academy.

Although daily life as a minority in cardiothoracic surgery may feel like a "battle" against unconscious and conscious biases, "Battle of the Sexes" is a charged title that contradicts our opinion. "Battle" implies opposing, hostile parties 